\title{
Dynamic Output Feedback Regulation for a Class of Nonlinear Systems*
}

\author{
J.-B. Pomet† R.M. Hirschorn and W.A. Cebuhar \\ Queen's University at Kingston \\ Kingston, Ontario K7L 3N6 Canada \\ January 31, 1992 \\ revised July 29, 1992, final version \\ To appear in : Mathematics of Control, Signals and Systems, vol. 6, 1993 \\ http://dx.doi.org/10.1007/BF01211742
}

\begin{abstract}
In this paper results are presented on the problem of regulating nonlinear systems by output feedback, using Lyapunov-based techniques. In all the cases considered here, we ask that the part of the state which is not measured enter linearly in the equations. Sufficient conditions for the global stabilization of the observed states via dynamic output feedback are obtained, assuming that such stabilization is possible using state feedback. Systems satisfying these conditions include a natural class of bilinear systems and systems which reduce to linear observable systems when the nonlinear terms in the measured states are removed. Some simple examples are included to illustrate our approach.
\end{abstract}

${ }^{*}$ This work was supported in part by the Natural Sciences and Engineering Research Council of Canada.

$\dagger^{\top}$ This author is now with Laboratoire d'Automatique de Nantes (URA C.N.R.S. 823), E.C.N., 44072 NANTES cedex 03, France; most of this work was done when he was with Queen's University. 


\section{Introduction}

This paper is concerned with the problem of output feedback regulation of nonlinear systems. Many authors have considered such problems, using various approaches. Some necessary and sufficient conditions for regulation via static output feedback are established in Tsinias and Kalouptsidis [TK], by extending previous results of Artstein [A] and Sontag [S1]. However, these conditions are not explicit and involve the existence of a special "control Lyapunov function." Furthermore, as it is well known, most feedback stabilizable systems are not static output feedback stabilizable (e.g. $\dot{x}_{1}=x_{2}, \dot{x}_{2}=u, y=x_{1}$ ).

For linear systems, a more general problem has been solved, among others, by Francis [F]. These results were extended to nonlinear systems by Isidori and Byrnes [IB], for the case in which some of the nonobserved dynamics evolve independently, as a so-called "exosystem". This exosystem is assumed to be Poisson stable. For the case in which the state of the exosystem is not available to the controller, local regulation results follow from detectability of the linear approximation of the combined system.

The most "natural" approach to output control is to try to build an observer. But for nonlinear systems the theory of observers is not well developed, and only partial results are known (see [I] sec 4.9). Even in the cases where it is possible to design an observer, it might not solve the problem of output control because the "separation principle", which is valuable in the linear case, does not hold in general. In [GK], Gauthier and Kupka have proved that this principle holds for a certain class of bilinear systems and very particular observers. In [V], a rather general condition is given for such a separation principle to hold. This was generalized by Tsinias $[\mathrm{T}]$, but the main result is local, and the situations considered here include many where it is not clear that an asymptotic observer can be built.

Output stabilization of a certain class of systems has been recently obtained, independently and by different methods, by Kanellakopoulos, Kokokovic and Morse in [KKM] and by Marino and Tomei in [MT1, MT2]. They consider output stabilization of a particular class of systems which are, roughly speaking, input-output linear up to output injection and are "minimum phase" in a rather strong sense (the zero-dynamics are also linear up to output injection). These systems have a common feature with those considered here, i.e. they are globally stabilizable by full-state feedback and (for proper coordinates in the state-space) the non-observed coordinates of the state appear linearly in the equations. The other assumptions considered in this paper (in particular A3 or A3', see section 2 below) are usually not satisfied in a straightforward manner.

Let us mention also some necessary and sufficient conditions given by Sontag in [S2] for the existence a stabilizing dynamic output controller, where "controller" is taken in a rather abstract sense; these conditions are not necessary here since they only characterize the case where all the state variables are required to converge whereas we only require that the output converges.

We follow an approach that does not involve explicitly building an observer. Our main assumptions are that regulation of the observed states is possible by full state feedback and that the nonobserved states enter the system equations linearly. Under these assumptions, together with some technical Lyapunov conditions, global results can be obtained. Our methods are an extension of those commonly used in adaptive stabilization (see, for example, [PBPJ]). It should be noted that nonlinear adaptive stabilization is a particular case of our problem, where the nonobserved states are constant (i.e. unknown parameters).

Some preliminary results using this approach were presented by the authors in $[\mathrm{CHP}]$, under rather restrictive technical assumptions. These results were extended by Praly $[\mathrm{P}]$ by using different Lyapunov techniques (leading to some "growth conditions" as assumptions) and taking advantage of a kind of weak observability.

In this paper, we present a dynamic controller similar to our original one $[\mathrm{CHP}]$, but the assumptions are greatly relaxed. We also use some observability conditions, as in $[\mathrm{P}]$. The results now apply to a much larger class of systems. In particular, we obtain output regulation for the 
same class of bilinear systems as in [GK], using a different controller.

\section{Problem Statement}

We consider a nonlinear system with input $u \in \mathbb{R}^{m}$ and output $y \in \mathbb{R}^{k}$ which admits a state-space representation in $\mathbb{R}^{n}(n \geq k)$ of the form

$$
\begin{aligned}
\dot{x}_{1} & =a_{1}\left(x_{1}, u\right)+A_{1}\left(x_{1}, u\right) x_{2} \\
\dot{x}_{2} & =a_{2}\left(x_{1}, u\right)+A_{2}\left(x_{1}, u\right) x_{2} \\
y & =h(x)=x_{1}
\end{aligned}
$$

with $x_{1} \in \mathbb{R}^{k}, x_{2} \in \mathbb{R}^{\ell},\left(x_{1}, x_{2}\right)^{T}=x \in \mathbb{R}^{n}$ and where $a_{1}, a_{2}, A_{1}$ and $A_{2}$ are, respectively, a $k$-vector, a $\ell$-vector, a $k \times \ell$-matrix and a $\ell \times \ell$-matrix smoothly depending on $x_{1}$ and $u$. The assumption contained in (1) is linearity of the equations with respect to the unmeasured states. We may also write

$$
\begin{aligned}
\dot{x} & =a\left(x_{1}, u\right)+A\left(x_{1}, u\right) x_{2} \\
y & =H x
\end{aligned}
$$

with

$$
a\left(x_{1}, u\right)=\left(\begin{array}{c}
a_{1}\left(x_{1}, u\right) \\
a_{2}\left(x_{1}, u\right)
\end{array}\right) \quad A\left(x_{1}, u\right)=\left(\begin{array}{c}
A_{1}\left(x_{1}, u\right) \\
A_{2}\left(x_{1}, u\right)
\end{array}\right) \quad H=\left(\begin{array}{ll}
I_{k} & 0
\end{array}\right)
$$

As explained above, we suppose that it is possible to design a full state feedback controller which regulates the output $x_{1}$, i.e. such that any solution of the closed-loop system, $x(t)=$ $\left(x_{1}(t), x_{2}(t)\right)$ is bounded and $x_{1}(t)$ goes to zero.

This is expressed by assumptions A1 or A1' below, via existence of some "Lyapunov-like" functions. We actually decompose the Lyapunov function into two parts $\left(V=V_{1}+V_{2}\right)$ where $V_{1}$ determines the design of the controller, and $V_{2}$ is assured to be non-increasing whatever the control is. This distinction is motivated by the fact that we will assume a special structure on $V_{1}$, which need not apply to all of $V$. In the case where the $x_{2}$ part of the system is an "exo-system" supposed to be Poisson stable, as in [IB], $V_{2}$ may be taken as a function of $x_{2}$. Motivated by various examples, we study our problem under two slightly different assumptions - $\mathrm{A} 1$ or $\mathrm{A} 1^{\prime}-$ which both express the ability to regulate using full-state feedback. Assumption A1' is somewhat less restrictive than A1 since (8) obviously implies (9). Assumption A1 lets us conclude that $x$ is bounded and $x_{1}$ goes to zero via the standard Lyapunov "second" theorem, whereas LaSalle's invariance principle (see $[\mathrm{L}]$ ) is needed to deduce this from assumption $\mathrm{A} 1^{\prime}$. It is often the case (as for bilinear systems, see section 4.2) that a simple non-strict Lyapunov function (i.e. satisfying (9) and not (8)) can be found; a more complicated one may very well exist that allows assumption A1 to be satisfied, but again, since we are going to impose some additional restrictions on $V_{1}$, we wish to keep the widest possible range in the choice of $V_{1}$.

Assumption A1 : There exist two continuously differentiable positive semidefinite functions $V_{1}$ and $V_{2}$ from $\mathbb{R}^{n}$ to $\mathbb{R}$ and a map $u_{\text {nom }}$ from $\mathbb{R}^{n}$ to $\mathbb{R}^{m}$ such that

1. The function $V$ from $\mathbb{R}^{n}$ to $\mathbb{R}$ defined by

$$
V(x)=V_{1}(x)+V_{2}(x)
$$

is proper (i.e. the preimage of a compact set is compact).

2. For any $x$ and $u$,

$$
\frac{\partial V_{2}}{\partial x}(x) \cdot\left[a\left(x_{1}, u\right)+A\left(x_{1}, u\right) x_{2}\right] \leq 0
$$


3. Defining the function $\rho$ by

$$
\rho(x)=-\frac{\partial V_{1}}{\partial x}(x)\left[a\left(x_{1}, u_{\mathrm{nom}}(x)\right)+A\left(x_{1}, u_{\mathrm{nom}}(x)\right) x_{2}\right],
$$

we have that

$$
\begin{aligned}
& \rho(x) \geq 0 \quad \forall x \in \mathbb{R}^{n} \\
& \rho(x)=0 \quad \Rightarrow \quad x_{1}=0
\end{aligned}
$$

Assumption $\mathbf{A 1}^{\prime}$ : This is the same as assumption A1 with (8) replaced by

$$
\left.\begin{array}{l}
\rho(x(t))=0 \quad \forall t \\
\frac{d x}{d t}(t)=a\left(x_{1}(t), u_{\text {nom }}(x(t))\right)+A\left(x_{1}(t), u_{\text {nom }}(x(t))\right) x_{2}(t) \quad \forall t
\end{array}\right\} \Rightarrow x_{1}(t)=0 \quad \forall t
$$

In order to obtain our global results, we will use the following somewhat restrictive hypothesis. Assumption A2 : $V_{1}$ has the form

$$
V_{1}(x)=U_{1}\left(x_{1}\right)+x_{2}^{T} L\left(x_{1}\right)+\frac{1}{2} x_{2}^{T} M x_{2}
$$

where $U_{1}$ and $L$ are smooth functions from $\mathbb{R}^{k}$ to $\mathbb{R}$ and from $\mathbb{R}^{k}$ to $\mathbb{R}^{\ell}$ respectively, and $M$ is a symmetric $\ell \times \ell$ real matrix.

Now, let us define the $n \times n$ matrices $N\left(x_{1}, x_{2}\right)$ and $E\left(x_{1}, x_{2}\right)$ by:

$$
\begin{gathered}
N\left(x_{1}, x_{2}\right)=\left(\begin{array}{cc}
0 & 0 \\
0 & Z\left(x_{1}, x_{2}\right)
\end{array}\right) \\
E\left(x_{1}, x_{2}\right)=\left(\begin{array}{cc}
0 & A_{1}\left(x_{1}, u_{\text {nom }}\left(x_{1}, x_{2}\right)\right) \\
0 & A_{2}\left(x_{1}, u_{\text {nom }}\left(x_{1}, x_{2}\right)\right)
\end{array}\right)
\end{gathered}
$$

where

$$
\begin{array}{r}
Z\left(x_{1}, x_{2}\right)=\frac{\partial L}{\partial x_{1}} A_{1}\left(x_{1}, u_{\mathrm{nom}}\left(x_{1}, x_{2}\right)\right)+\left[\frac{\partial L}{\partial x_{1}} A_{1}\left(x_{1}, u_{\mathrm{nom}}\left(x_{1}, x_{2}\right)\right)\right]^{T} \\
+M A_{2}\left(x_{1}, u_{\mathrm{nom}}\left(x_{1}, x_{2}\right)\right)+A_{2}^{T}\left(x_{1}, u_{\mathrm{nom}}\left(x_{1}, x_{2}\right)\right) M
\end{array}
$$

Our third assumption is a kind of weak observability. Assumption A3 is the weakest version, while assumption $\mathrm{A} 3^{\prime}$ is stronger. An even stronger $\mathrm{A} 3^{\prime \prime}$ will allow us to get a converging estimate of the unmeasured states.

Assumption A3 : There exist a constant positive definite $n \times n$ matrix $Q$ and an $n \times k$ matrix $K\left(x_{1}, x_{2}\right)$ smoothly depending on $x=\left(x_{1}, x_{2}\right)$ such that, for any $x$,

$$
Q\left(E\left(x_{1}, x_{2}\right)-K\left(x_{1}, x_{2}\right) H\right)+\left(E\left(x_{1}, x_{2}\right)-K\left(x_{1}, x_{2}\right) H\right)^{T} Q+N\left(x_{1}, x_{2}\right) \leq 0
$$

in the sense of symmetric matrices.

Assumption $\mathbf{A 3}^{\prime}$ : There exist a constant positive definite $n \times n$ matrix $Q$ and an $n \times k$ matrix $K\left(x_{1}, x_{2}\right)$ smoothly depending on $x=\left(x_{1}, x_{2}\right)$ such that, for any $x=\left(x_{1}, x_{2}\right)$, the matrix (14) is negative definite for $x_{1} \neq 0$, i.e.

$$
Q\left(E\left(x_{1}, x_{2}\right)-K\left(x_{1}, x_{2}\right) H\right)+\left(E\left(x_{1}, x_{2}\right)-K\left(x_{1}, x_{2}\right) H\right)^{T} Q+N\left(x_{1}, x_{2}\right) \leq-\alpha\left(x_{1}, x_{2}\right) I
$$

for a certain $\alpha\left(x_{1}, x_{2}\right)$ such that

$$
\alpha\left(x_{1}, x_{2}\right) \geq 0 \forall x_{1}, x_{2} \text { and } \alpha\left(x_{1}, x_{2}\right)=0 \Longrightarrow x_{1}=0
$$


Assumption $\mathbf{A 3}^{\prime \prime}$ : There exist a constant positive definite $n \times n$ matrix $Q$ and an $n \times k$ matrix $K\left(x_{1}, x_{2}\right)$ smoothly depending on $x=\left(x_{1}, x_{2}\right)$ such that, for any $x$, is negative definite for $x_{1} \neq 0$, i.e.

$$
Q\left(E\left(x_{1}, x_{2}\right)-K\left(x_{1}, x_{2}\right) H\right)+\left(E\left(x_{1}, x_{2}\right)-K\left(x_{1}, x_{2}\right) H\right)^{T} Q+N\left(x_{1}, x_{2}\right) \leq-\alpha I
$$

with $\alpha$ a positive constant.

\section{Remarks:}

- These assumptions are somehow similar to assumption $\mathrm{D}$ in $[\mathrm{P}]$ in the sense that they open up the possibility to use a gain $K$ to counteract some possibly unstable terms (here, $N$ ).

- If the matrix $Q$ were not required to be constant, a sufficient condition for $\mathrm{A} 3^{\prime}$ would be that the pair $(E(x), H)$ be observable for any $x$ (and a sufficient condition for A3 that its unobservable modes be marginally stable). The fact that we ask $Q$ to be constant makes it much more difficult to give reasonable sufficient conditions in terms of the "family of pairs" $(E(x), H)$ indexed by $x$. Our Example 3 in Section 4.3 displays a very particular situation where we may extend our methods to work without the requirement that $Q$ be constant.

\section{The controller and main results}

\subsection{The controller}

We shall use the following notations:

- The dynamic controller we are designing has state $\widehat{x}=\left(\widehat{x}_{1}, \widehat{x}_{2}\right)$.

- $\widetilde{x}_{1}, \widetilde{x}_{2}, \widetilde{x}$ stand for $x_{1}-\widehat{x}_{1}, x_{2}-\widehat{x}_{2}, x-\widehat{x}$, respectively.

- A hat on functions depending on $u$ or $x_{2}$ indicates it is evaluated with $\widehat{x}_{2}$ substituted for $x_{2}$ and for $u=u_{\text {nom }}\left(x_{1}, \widehat{x}_{2}\right)$ e.g.

$$
\begin{gathered}
\widehat{A}_{1} \text { stands for } A_{1}\left(x_{1}, u_{\text {nom }}\left(x_{1}, \widehat{x}_{2}\right)\right) \\
\widehat{a}_{2} \text { stands for } a_{2}\left(x_{1}, u_{\text {nom }}\left(x_{1}, \widehat{x}_{2}\right)\right) \\
\frac{\partial V_{1}}{\partial x_{1}} \text { stands for } \frac{\partial V_{1}}{\partial x_{1}}\left(x_{1}, \widehat{x}_{2}\right) \\
\widehat{K} \text { stands for } K\left(x_{1}, \widehat{x}_{2}\right)
\end{gathered}
$$

The dynamic controller we propose is then :

$$
\begin{aligned}
u & =u_{\text {nom }}\left(x_{1}, \hat{x}_{2}\right) \\
\dot{\hat{x}} & =\widehat{a}+\widehat{A} \hat{x}_{2}+\widehat{K} \tilde{x}_{1}+Q^{-1}\left(\begin{array}{c}
0 \\
P\left(x_{1}, \hat{x}_{2}\right)
\end{array}\right)
\end{aligned}
$$

with

$$
\begin{aligned}
P\left(x_{1}, \hat{x}_{2}\right) & =\frac{\partial L}{\partial x_{1}}\left(\widehat{a}_{1}+\widehat{A}_{1} \hat{x}_{2}\right)+M\left(\widehat{a}_{2}+\widehat{A}_{2} \hat{x}_{2}\right) \\
& +\widehat{A}_{1}^{\mathrm{T}}\left(\frac{\partial U_{1}}{\partial x_{1}}+\frac{\partial L}{\partial x_{1}} \hat{x}_{2}\right)^{\mathrm{T}}+\widehat{A}_{2}^{\mathrm{T}}\left(L+M \hat{x}_{2}\right) .
\end{aligned}
$$

where $Q$ and $K$ are given by assumption $\mathrm{A} 3$ or $\mathrm{A} 3^{\prime}$ depending which one is met.

The idea behind this design is to consider $V_{1}\left(x_{1}, x_{2}\right)+\frac{1}{2} \tilde{x}^{\mathrm{T}} Q \tilde{x}$ as a Lyapunov function candidate and design $\dot{\hat{x}}$ so that it decreases. This is done by canceling all the terms in its derivative other than $-\rho\left(x_{1}, \hat{x}_{2}\right)$, which appears rather naturally. For the details, see the proof of Lemma 1 below. 


\subsection{Regulation results}

We are stating here our main results concerning the properties of the closed-loop when using our dynamic regulator.

Theorem 1 Under assumptions A1, A2 and A3, or A1', A2 and A3', the dynamic controller (18)-(19) achieves the following property for the closed-loop system: for any initial conditions, the state $\left(x_{1}(t), x_{2}(t), \widehat{x}_{1}(t), \widehat{x}_{2}(t)\right)$ is bounded, and $x_{1}(t) \rightarrow 0$. Moreover, if assumption A3" is met, then $x_{1}(t)-\widehat{x}_{1}(t)$ and $x_{2}(t)-\widehat{x}_{2}(t)$ converge to 0 .

The proof of this theorem is based on the following lemma.

Lemma 1 The derivative of the function

$$
W_{1}(x, \hat{x})=V_{1}\left(x_{1}, x_{2}\right)+\frac{1}{2} \tilde{x}^{\mathrm{T}} Q \tilde{x}
$$

along the solutions of the closed-loop system obtained with controller (18)-(19) is given by :

$$
\dot{W}_{1}=-\rho\left(x_{1}, \hat{x}_{2}\right)+\frac{1}{2} \tilde{x}^{\mathrm{T}}\left[\widehat{N}+Q(\widehat{E}-\widehat{K} H)+(\widehat{E}-\widehat{K} H)^{\mathrm{T}} Q\right] \tilde{x} .
$$

Proof : We have, from (21),

$$
\dot{W}_{1}(x, \hat{x})=\dot{V}_{1}\left(x_{1}, x_{2}\right)+\tilde{x}^{\mathrm{T}} Q(\dot{x}-\dot{\hat{x}}) .
$$

Now, on one hand,

$$
\dot{V}_{1}=\frac{\partial V_{1}}{\partial x_{1}}\left(\widehat{a}_{1}+\widehat{A}_{1} x_{2}\right)+\frac{\partial V_{1}}{\partial x_{2}}\left(\widehat{a}_{2}+\widehat{A}_{2} x_{2}\right)
$$

which gives, since

$$
\begin{aligned}
& \frac{\widehat{\partial V_{1}}}{\partial x_{1}}\left(\widehat{a}_{2}+\widehat{A}_{2} \hat{x}_{2}\right)+\frac{\widehat{\partial V_{1}}}{\partial x_{2}}\left(\widehat{a}_{2}+\widehat{A}_{2} \hat{x}_{2}\right)=-\rho\left(x_{1}, \hat{x}_{2}\right), \\
\dot{V}_{1}= & -\rho\left(x_{1}, \hat{x}_{2}\right) \\
+ & \left(\frac{\partial V}{\partial x_{1}}-\frac{\widehat{\partial V}}{\partial x_{1}}\right)\left(\widehat{a}_{1}+\widehat{A}_{1} \hat{x}_{2}\right)+\left(\frac{\partial V_{1}}{\partial x_{2}}-\frac{\widehat{\partial V_{1}}}{\partial x_{2}}\right)\left(\widehat{a}_{2}+\widehat{A}_{2} \hat{x}_{2}\right) \\
& +\left[\frac{\partial V_{1}}{\partial x_{1}} \widehat{A}_{1}+\frac{\partial V_{1}}{\partial x_{2}} \widehat{A}_{2}\right] \widetilde{x}_{2} \\
& +\left[\left(\frac{\partial V_{1}}{\partial x_{1}}-\frac{\partial V_{1}}{\partial x_{1}}\right) \widehat{A}_{1}+\left(\frac{\partial V_{1}}{\partial x_{2}}-\frac{\partial V_{1}}{\partial x_{2}}\right) \widehat{A}_{2}\right] \widetilde{x}_{2} \\
= & -\rho\left(x_{1}, \hat{x}_{2}\right) \\
& +\widetilde{x}_{2}^{\mathrm{T}}\left[\frac{\partial L}{\partial x_{1}}\left(\widehat{a}_{1}+\widehat{A}_{1} \hat{x}_{2}\right)+M\left(\widehat{a}_{2}+\widehat{A}_{2} \hat{x}_{2}\right)\right] \\
& +\left[\left(\frac{\partial U_{1}}{\partial x_{1}}+\hat{x}_{2}^{\mathrm{T}} \frac{\partial L}{\partial x_{1}}\right) \widehat{A}_{1}+\left(L \hat{x}^{\mathrm{T}}+\hat{x}_{2}^{\mathrm{T}} M\right) \widehat{A}_{2}\right] \widetilde{x}_{2} \\
+ & \widetilde{x}_{2}^{\mathrm{T}}\left[\frac{\partial L}{\partial x_{1}} \widehat{A}_{1}+M \widehat{A}_{2}\right] \widetilde{x}_{2} \\
+ & \rho\left(x_{1}, \hat{x}_{2}\right)+\widetilde{x}_{2}^{\mathrm{T}} \widehat{P} \\
& \frac{1}{2} \widetilde{x}_{2}^{\mathrm{T}}\left[\frac{\partial L}{\partial x_{1}} \widehat{A}_{1}+\widehat{A}_{1}^{\mathrm{T}} \frac{\partial L}{\partial x_{1}}+M \widehat{A}_{2}+\widehat{A}_{2}^{\mathrm{T}} M\right] \widetilde{x}_{2} \\
&
\end{aligned}
$$


where $\widehat{P}$ stands for $P\left(x_{1}, \hat{x}_{2}\right)$ (see $\left.(20)\right)$. On the other hand, from (1) and (21),

$$
\begin{aligned}
\dot{x}-\dot{\hat{x}} & =\widehat{A} \widetilde{x}_{2}-\widehat{K} \widetilde{x}_{1}-Q^{-1}\left(\begin{array}{c}
0 \\
P\left(x_{1}, \hat{x}_{2}\right)
\end{array}\right) \\
& =(\widehat{E}-\widehat{K} H) \tilde{x}-Q^{-1}\left(\begin{array}{c}
0 \\
P\left(x_{1}, \hat{x}_{2}\right)
\end{array}\right) .
\end{aligned}
$$

Clearly (27) and (22) imply (21).

Proof of the theorem: We use the result of Lemma 1. Let us define the function $W$ of the closed-loop state $(x, \widehat{x})$ by

$$
W(x, \widehat{x})=W_{1}(x, \widehat{x})+V_{2}(x)
$$

From (21) and (4), $W$ is a proper function of $(x, \widehat{x})$ and, from $(22)$ and (5), we have:

$$
\dot{W}(x, \widehat{x}) \leq-\rho\left(x_{1}, \widehat{x}_{2}\right)+\frac{1}{2} \widetilde{x}^{\mathrm{T}}\left[\widehat{N}+Q(\widehat{E}-\widehat{K} H)+(\widehat{E}-\widehat{K} H)^{T} Q\right] \widetilde{x}
$$

This proves that on any solution of the closed-loop system, $W$ decreases. Therefore all the solutions are bounded.

We may now apply LaSalle's invariance principle: $(x(t), \widehat{x}(t))$ goes to the largest invariant subset of the set of $(x, \widehat{x})$ 's where the right-hand side of $(28)$ is zero.

If $\mathrm{A} 3$ is met, (28) reduces to $\dot{W} \leq-\rho\left(x_{1}, \widehat{x}_{2}\right)$. Therefore the invariant set is defined by $\rho\left(x_{1}, \widehat{x}_{2}\right)=0$ which, under assumption A1, implies $x_{1}=0$.

Now suppose that $\mathrm{A}^{\prime}$ and $\mathrm{A} 3^{\prime}$ are met. In this case, making the right-hand side of (28) equal to zero implies $\rho\left(x_{1}, \widehat{x}_{2}\right)=0$ and $\alpha\left(x_{1}, \widehat{x}_{2}\right)\|\tilde{x}\|^{2}=0$. If $\alpha\left(x_{1}, \widehat{x}_{2}\right)$ is identically zero on a solution of the closed-loop, then $x_{1}=0$ on this solution by assumption A3'. On the other hand, if $\alpha\left(x_{1}, \widehat{x}_{2}\right)>0$, then $\|\tilde{x}\|=0$, hence $\widehat{x}_{2}=x_{2}$, and now $\mathrm{A} 1^{\prime}$ implies $x_{1}=0$.

Finally, if $\mathrm{A} 3^{\prime \prime}$ is met (i.e. if $\alpha$ is a positive constant), we always have $\|\tilde{x}\|=0$ for the invariant set. Therefore $\widehat{x}_{1}(t) \longrightarrow x_{1}(t)$ and $\widehat{x}_{2}(t) \longrightarrow x_{2}(t)$ as stated.

A closer examination of the proof of Theorem 1 indicates that, instead of assumptions A1 and $\mathrm{A} 3$ or $\mathrm{A} 1^{\prime}$ and $\mathrm{A} 3^{\prime}$, there is a very natural assumption which allows one to get the same regulation result :

Assumption A4 : Assumption A3 holds as well as assumption A1 with (8) replaced by :

$$
\left.\begin{array}{l}
\eta(x(t), \widehat{x}(t))=0 \quad \forall t \\
(x(t), \widehat{x}(t)) \text { is a trajectory for the combined }
\end{array}\right\} \Longrightarrow x_{1}(t)=0 \forall t,
$$

where $\eta$ is defined by

$$
\eta(x, \widehat{x})=\rho\left(x_{1}, \widehat{x}_{2}\right)-\frac{1}{2} \widetilde{x}^{\mathrm{T}}\left[\widehat{N}+Q(\widehat{E}-\widehat{K} H)+(\widehat{E}-\widehat{K} H)^{T} Q\right] \widetilde{x}
$$

This assumption is obviously implied either by assumptions A1 and A3 or by assumptions A1' and $\mathrm{A} 3^{\prime}$. It is rather implicit (since it is given in terms of the solutions of the closed-loop system), and the corresponding theorem, stated below, is quite straightforward. However, when working on examples, one realizes that in some interesting cases (C.f. Ex. 2), A4 is met (and our controller therefore yields global regulation) whereas neither of the sets of assumptions A1-A3 or A1'-A3' are met. This is the motivation for the following theorem :

Theorem 2 Under assumptions A2 and A4 the dynamic controller (18)-(19) achieves the following property for the closed-loop system: for any initial conditions, the state $\left(x_{1}(t), x_{2}(t), \widehat{x}_{1}(t), \widehat{x}_{2}(t)\right)$ is bounded and $x_{1}(t)$ converges to 0 . 
Proof : We proceed as in Theorem 1 to obtain

$$
\dot{W}(x, \widehat{x}) \leq-\eta(x, \widehat{x})
$$

Then assumption A4 is exactly what is needed to conclude $x_{1} \longrightarrow 0$ from LaSalle's invariance principle.

\subsection{Reduced order controller}

In this section, we present a result that was obtained in $[\mathrm{CHP}]$ under more restrictive assumptions. Those assumptions actually imply the ones we are making in this paper, so it is possible to simply use our present controller for that case. However, in order to obtain a simpler controller (of reduced order), as in [CHP], we consider a special case of assumption A3. The positive definite matrix $Q$ in $\mathrm{A} 3$ or $\mathrm{A}^{\prime}$ is replaced by $\left[\begin{array}{cc}0 & 0 \\ 0 & Q_{2}\end{array}\right]$ with $Q_{2}>0$ and $K$ is assumed to be zero.

Assumption $\mathbf{A 3}^{\prime \prime \prime}$ : There exists a (constant) positive definite $\ell \times \ell$ matrix $Q_{2}$ such that, for any $x$,

$$
Q_{2} A_{2}\left(x_{1}, u_{\text {nom }}\left(x_{1}, x_{2}\right)\right)+A_{2}\left(x_{1}, u_{\text {nom }}\left(x_{1}, x_{2}\right)\right)^{T} Q_{2}+Z \leq 0
$$

where $Z$ is defined by equation (13).

The reduced order dynamic controller is then:

$$
\begin{aligned}
u & =u_{\text {nom }}\left(x_{1}, \widehat{x}_{2}\right) \\
\dot{\hat{x}}_{2} & =\widehat{a}_{2}+\widehat{A}_{2} \widehat{x}_{2}+Q_{2}^{-1} P\left(x_{1}, \widehat{x}_{2}\right)
\end{aligned}
$$

where $P$ is given by (20). Then Theorem 1 becomes:

Theorem 3 Under assumptions A1, A2 and A3'"', the dynamic controller (33)-(34) achieves the following property for the closed-loop system: for any initial conditions, the state $\left(x_{1}(t), x_{2}(t), \widehat{x}_{2}(t)\right)$ is bounded, and $x_{1}(t)$ converges to 0 .

The proof is essentially the same as before and is given in $[\mathrm{CHP}]$.

\subsection{Complete stabilization}

Although this paper is more oriented towards output regulation (regulation of $x_{1}$ ), an extension of the above results to regulation of the whole state $x=\left(x_{1}, x_{2}\right)$ is possible, under the assumption that the full-state control $u_{\text {nom }}$ not only regulates $x_{1}$, but $\left(x_{1}, x_{2}\right)$, i.e. it is a stabilizing feedback control for the system (1):

Assumption $\mathbf{A 1}^{\prime \prime}$ : This is assumption $A 1^{\prime}$, with (9) replaced by

$$
\left.\begin{array}{l}
\rho(x(t))=0 \quad \forall t \\
\frac{d x}{d t}(t)=a\left(x_{1}(t), u_{\mathrm{nom}}(x(t))\right)+A\left(x_{1}(t), u_{\mathrm{nom}}(x(t))\right) x_{2}(t) \quad \forall t
\end{array}\right\} \Rightarrow x(t)=0 \quad \forall t
$$

We have the following result:

Theorem 4 : Under assumptions A1", A2 and A3", the dynamic controller (18)-(19) is such that $(x, \widehat{x})=(0,0)$ is a globally asymptotically stable equilibrium of the closed-loop system.

Remark: A somewhat less restrictive version of the above theorem can be obtained for the case where (17) does not hold with a positive constant $\alpha$, but for a positive function $\alpha(x)$-as in assumption $\mathrm{A} 3^{\prime}$ - with the property -stronger than (15) in assumption A3'- that $\alpha(x)=0 \Longrightarrow x=$ 0 ; in that case, we have to suppose, in addition (for a proper application of LaSalle's Principle), that $\left(A_{2}\left(0, u_{\text {nom }}(0,0)\right), A_{1}\left(0, u_{\text {nom }}(0,0)\right)\right)$ is an observable pair (i.e. the linear approximation to system (1) around $x_{1}=0$ and $u=u_{\text {nom }}(0,0)$ is observable). 


\section{Applications}

\subsection{Systems Strictly Linear in the Unobserved States}

By "strictly linear", we mean that system (1) is linear with respect to the unobserved part of the state, $x_{2}$, with a constant regressor, i.e. that the matrix $A\left(x_{1}, u\right)$ in the state equation $(2)$ is constant. If, in addition, the linear approximation is detectable then assumption $\mathrm{A} 3^{\prime \prime}$ is equivalent to a simple growth condition on the "off-diagonal terms" in the Lyapunov function $V_{1}$.

Proposition 1 Suppose that the matrix $A=\left(A_{1}, A_{2}\right)^{\mathrm{T}}$ defining system (1) is constant, and the linear approximation of (1) around $(x, u)=(0,0)$ is observable. Let $V_{1}$ be a function satisfying assumption A2 for certain $U_{1}, L$ and $M$. If there exists a positive constant $\alpha$ such that, for all $x_{1}$,

$$
\frac{\partial L}{\partial x_{1}}\left(x_{1}\right) A_{1}+A_{1}^{T} \frac{\partial L^{T}}{\partial x_{1}}\left(x_{1}\right) \leq \alpha I,
$$

then assumption $A 3^{\prime \prime}$ is met.

This proposition allows us to apply one of the theorems above if, in addition, one of the full state regulation assumptions ( $\mathrm{A} 1, \mathrm{~A} 1^{\prime}$ or $\left.\mathrm{A} 1^{\prime \prime}\right)$ is met.

Let us prove this proposition. Condition (36) and $A_{2}$ constant imply that the matrix $N\left(x_{1}, x_{2}\right)$ defined by (11) and (13) is bounded. Thus there exists a constant $\beta$ such that $N\left(x_{1}, x_{2}\right) \leq \beta I$ for all $x_{1}, x_{2}$, and hence $\mathrm{A} 3^{\prime \prime}$ will hold if there exists a constant positive definite $n \times n$ matrix $Q$ and an $\ell \times n$ matrix $K\left(x_{1}, x_{2}\right)$ smoothly depending on $x=\left(x_{1}, x_{2}\right)$ such that for any $x$,

$$
Q\left(E\left(x_{1}, x_{2}\right)-K\left(x_{1}, x_{2}\right) H\right)+\left(E\left(x_{1}, x_{2}\right)-K\left(x_{1}, x_{2}\right) H\right)^{T} Q \leq-2|\beta| I .
$$

If the linear approximation to our system is detectable then such a $K$ and $Q$ exist. To see this, recall that the linear approximation to $\dot{x}=f(x, u) ; y=h(x)$ about $(x, u)=(0,0)$ is the time invariant linear system $(F, G, H)$ where

$$
F=\left.\frac{\partial f}{\partial x}\right|_{x=0, u=0}, G=\left.\frac{\partial f}{\partial u}\right|_{x=0, u=0}, H=\left.\frac{\partial h}{\partial x}\right|_{x=0} .
$$

The linear approximation to system (1) when $A$ is constant has

$$
F=\left[\left.\frac{\partial a}{\partial x_{1}}\right|_{x=0, u=0} A\right], G=\left.\frac{\partial a}{\partial u}\right|_{x_{1}=0, u=0}, H=\left[\begin{array}{ll}
I & 0
\end{array}\right] .
$$

If this system is detectable, there exists an $n \times \ell$ matrix $K$ such that $F-K H$, or equivalently, $E-K H$ has its poles strictly in the left-half plane, where $E=\left[\begin{array}{ll}0 & A\end{array}\right]$.

In this case there exists a unique positive definite matrix $Q$ solving

$$
Q(E-K H)+(E-K H)^{T} Q \leq-2|\beta| I
$$

(cf. $[\mathrm{B}])$, hence

$$
Q(E-K H)+(E-K H)^{T} Q+N \leq-|\beta| I
$$

and condition $\mathrm{A} 3^{\prime \prime}$ is satisfied.

Remark: Suppose that the system (1) is a time-invariant linear system which satisfies assumption A1 with $u_{\text {nom }}$ linear and $V_{1}$ quadratic. Then assumption A2 holds with $L\left(x_{1}\right)$ linear (and hence the growth condition (36) holds as well), and assumption $\mathrm{A} 3{ }^{\prime \prime}$ follows if, in addition, the system is detectable. 


\subsection{Bilinear systems}

Consider the bilinear system

$$
\begin{aligned}
\dot{x} & =F x+\sum_{k=1}^{m} u_{k} G_{k} x \\
y & =H x=x_{1}
\end{aligned}
$$

where the observed states are the $k$ first components of $x$. We shall prove that it is possible to use Theorem 4 to stabilize the origin with the only measurement of $x_{1}$, under the following assumptions:

1. First, we ask that the system has a controllable linear approximation (i.e. the "ad-condition" is satisfied, see $[\mathrm{JQ}]$ ) everywhere except at zero:

$$
\forall x \neq 0, \operatorname{Span}\left\{\left(a d_{F}^{j} G_{k}\right) x \mid j \geq 0, k=1 \ldots m\right\}=\mathbb{R}^{n}
$$

where the ad operator is the one corresponding to matrix commutators:

$a d_{F}^{0} G=G$ and $a d_{F}^{j+1} G=F\left(a d_{F}^{j} G\right)-\left(a d_{F}^{j} G\right) F$.

2. System (37) also has to be observable for small inputs:

$$
\operatorname{Rank}\left(H, H F, H F^{2}, \ldots, H F^{n-1}\right)=n
$$

3. Finally, we ask that the system be naturally (with a zero control) dissipative or conservative; namely we suppose that all the eigenvalues of $F$ have nonpositive real parts and that the Jordan blocks corresponding to the imaginary ones be diagonal.

Proposition 2 If points 1, 2 and 3 above are satisfied then it is possible to satisfy the assumptions of Theorem 4, and the controller (18)-(19) is then a stabilizing output feedback controller for system (37).

Let us prove this proposition, i.e. explain how to build the appropriate $u_{\text {nom }}, V_{1}$ and $V_{2}$ for the system (37). Points 1 and 3 allow us to derive a stabilizing full state control law for system (38), following the idea of $[\mathrm{JQ}]$ (see also $[\mathrm{NV}]$ ) : point 3 implies the existence of a symmetric, positive definite matrix $\Gamma$ such that

$$
\Gamma F+F^{T} \Gamma \leq 0
$$

Now take

$$
V_{1}(x)=x^{T} \Gamma x
$$

and define $u_{\text {nom }}$ by :

$$
u_{\mathrm{nom}, \mathrm{k}}(x)=-\frac{\lambda}{\sqrt{1+\left(x^{T} \Gamma G_{k} x\right)^{2}}} x^{T} \Gamma G_{k} x,
$$

where $\lambda$ is a positive constant. This yields

$$
\dot{V}_{1}=-\rho(x)+x^{\mathrm{T}}\left(\Gamma F+F^{\mathrm{T}} \Gamma\right) x \leq 0
$$

with

$$
\rho(x)=\lambda \sum_{k=1}^{m} \frac{\left(x^{T} \Gamma G_{k} x\right)^{2}}{\sqrt{1+\left(x^{T} \Gamma G_{k} x\right)^{2}}} .
$$


The reason for the coefficient $\left(1+\left(x^{T} \Gamma G_{k} x\right)^{2}\right)^{\frac{1}{2}}$ is to obtain a bounded control: (42) implies that

$$
\left|u_{\text {nom }, \mathrm{k}}(x)\right|<\lambda \quad \forall x \in \mathbb{R}^{n} .
$$

Suppose that $x(t)$ is a system trajectory such that the right-hand side of (43) is identically zero. Then $x^{\mathrm{T}}(t)\left(\Gamma F+F^{\mathrm{T}} \Gamma\right) x(t)=0$ for all $t$. Since $\Gamma F+F^{\mathrm{T}} \Gamma$ is negative semidefinite, it follows that $x^{\mathrm{T}}(t)\left(\Gamma F+F^{\mathrm{T}} \Gamma\right)=0$, and thus $x^{\mathrm{T}}(t) \Gamma F=-x^{\mathrm{T}}(t) F^{\mathrm{T}} \Gamma$ for all $t$. Using this fact and point 1 above one can check (see $[\mathrm{JQ}]$, or $[\mathrm{NV}]$, for some details) that the following is true for any function $x(t)$ :

$$
\left.\begin{array}{rr}
x(t)^{T} \Gamma G_{k} x(t)=0 \quad \forall k=1, \ldots, m & \forall t \\
\dot{x}(t)=F x(t) & \forall t
\end{array}\right\} \Longrightarrow x(t)=0, \forall t .
$$

From (44), $\rho(x)$ is zero if and only if all the $x^{\mathrm{T}} \Gamma G_{k} x$ 's are zero. As in [JQ], we can conclude that, from (46), (43) and the fact that $V_{1}$ is proper, $x=0$ is an asymptotically stable equilibrium of the closed loop obtained with the full state feedback $u=u_{\text {nom }}(x)$.

Under the additional assumption (observability) contained in point 2, we are able to derive an output feedback controller. Note that Gauthier and Kupka have given in [GK] an output controller which yields stabilization in the very same situation; they use quite a different method, explicitly based on the convergence of a nonlinear observer, and the controller obtained is different from ours.

Let us prove that we are in the situation of Theorem 4. First of all, system (37) is of the form (1) with

$$
\begin{aligned}
& a_{1}\left(x_{1}, u\right)=F_{11} x_{1}+\sum u_{k} G_{k, 11} x_{1} \\
& a_{2}\left(x_{1}, u\right)=F_{21} x_{1}+\sum u_{k} G_{k, 21} x_{1} \\
& A_{1}\left(x_{1}, u\right)=F_{12}+\sum u_{k} G_{k, 12} \\
& A_{2}\left(x_{1}, u\right)=F_{22}+\sum u_{k} G_{k, 22}
\end{aligned}
$$

where $F_{11}, F_{12}, F_{21}, F_{22}$ are defined by

$$
F=\left(\begin{array}{ll}
F_{11} & F_{12} \\
F_{21} & F_{22}
\end{array}\right) \quad G_{k}=\left(\begin{array}{ll}
G_{k, 11} & G_{k, 21} \\
G_{k, 12} & G_{k, 22}
\end{array}\right)
$$

Assumption $\mathrm{A} 1^{\prime \prime}$ is met with $V_{1}$ defined by (41), $V_{2}=0, u_{\text {nom }}$ defined by (42) and $\rho$ defined by (44) : points 1 and 2 in $A 1^{\prime \prime}$ are obvious and point 3 is a consequence of (46). Assumption A2 is obviously met with

$$
U_{1}\left(x_{1}\right)=x_{1}^{T} \Gamma_{11} x_{1}, \quad L\left(x_{1}\right)=2 \Gamma_{12} x_{1}, \quad M=\Gamma_{22} .
$$

Let us prove that assumption $\mathrm{A} 3^{\prime \prime}$ is met for $\lambda$ small enough. From (39), there exists $\bar{K}$ such that the matrix $F-\bar{K} H$ is Hurwitz. On the other hand, $N$ is, in the present case, a constant matrix, as seen from (11) and (49). Therefore there exists a positive definite matrix $Q$ such that, for instance

$$
Q(F-\bar{K} H)+(F-\bar{K} H)^{T} Q=-N-2 \alpha I
$$

with $\alpha$ a positive constant. But assumption $A 3^{\prime \prime}$ requires $E$ in place of $F$ in (50).

From (47) and (12),

Then, with

$$
E(x)=F+\left(\begin{array}{cc}
-F_{11} & \sum u_{\mathrm{nom}, \mathrm{k}}(x) G_{k, 12} \\
-F_{21} & \sum u_{\mathrm{nom}, \mathrm{k}}(x) G_{k, 22}
\end{array}\right) .
$$

$$
K(x)=\bar{K}-\left(\begin{array}{c}
F_{11}+\sum u_{\mathrm{nom}, \mathrm{k}}(x) G_{k, 11} \\
F_{21}+\sum u_{\mathrm{nom}, \mathrm{k}}(x) G_{k, 21}
\end{array}\right)
$$


it follows that

$$
Q(E-K H)+(E-K H)^{T} Q+N=-2 \alpha I-\sum u_{\mathrm{nom}, \mathrm{k}}(x)\left(Q G_{k}+G_{k}^{T} Q\right)
$$

From (45), it is clear that, for a choice of the constant $\lambda$ small enough, the right-hand side of (51) is smaller than $-\alpha I$, and therefore that assumption $\mathrm{A} 3{ }^{\prime \prime}$ is met.

\subsection{Examples}

Example 1. Let us consider the system

$$
\begin{aligned}
\dot{z}_{1} & =\phi\left(z_{1}\right) z_{2}+u \\
\dot{z}_{2} & =-z_{3} \\
\dot{z}_{3} & =z_{2} \\
y & =z_{1},
\end{aligned}
$$

where $\phi$ is a smooth real function. It is clearly of the form (1) with $x=\left(z_{1}, z_{2}, z_{3}\right), x_{1}=z_{1}$, $x_{2}=\left(z_{2}, z_{3}\right)$.

This is a typical illustration of the problem considered in [IB], $\left(z_{2}, z_{3}\right)$ being the state of the "exo-system", clearly Poisson stable since it is an harmonic oscillator. Let us give our controller for this example and compare it with the techniques from [IB].

If we use

$$
\begin{aligned}
u_{\text {nom }}\left(z_{1}, z_{2}, z_{3}\right) & =-\phi\left(z_{1}\right) z_{2}-z_{1} \\
V_{1}(x) & =\frac{1}{2} z_{1}^{2} \\
V_{2}(x) & =\frac{1}{2}\left(z_{2}^{2}+z_{3}^{2}\right)
\end{aligned}
$$

then assumption A1 is met with $\rho(x)=z_{1}^{2}$. Clearly assumption A2 is also satisfied, with $L=$ $M=0$. Computing $E$ according to (12), we obtain

$$
E=\left[\begin{array}{ccc}
0 & \phi\left(z_{1}\right) & 0 \\
0 & 0 & -1 \\
0 & 1 & 0
\end{array}\right]
$$

then

$$
E-K\left(z_{1}, z_{2}\right) H=\left[\begin{array}{ccc}
-k_{1} & \phi\left(z_{1}\right) & 0 \\
-k_{2} & 0 & -1 \\
-k_{3} & 1 & 0
\end{array}\right]
$$

and

$$
(E-K H)+(E-K H)^{T}=\left[\begin{array}{ccc}
-2 k_{1} & -k_{2}+\phi\left(z_{1}\right) & -k_{3} \\
-k_{2}+\phi\left(z_{1}\right) & 0 & 0 \\
-k_{3} & 0 & 0
\end{array}\right]
$$

Then, since $N=0$, assumption A3 is satisfied, with $Q=I$, if we take $k_{1}=1, k_{2}=\phi\left(z_{1}\right)$, and $k_{3}=0$.

In this case, our dynamic controller takes the form

$$
u=u_{\mathrm{nom}}\left(z_{1}, \widehat{z}_{2}, \widehat{z}_{3}\right)=-\phi\left(z_{1}\right) \widehat{z}_{2}-z_{1}
$$

where 


$$
\begin{aligned}
& \dot{\widehat{z}}_{1}=-\widehat{z}_{1} \\
& \dot{\widehat{z}}_{2}=-\widehat{z}_{3}+\left(2 z_{1}^{2}-\widehat{z}_{1}\right) \phi\left(z_{1}\right) \\
& \dot{\widehat{z}}_{3}=\widehat{z}_{2}
\end{aligned}
$$

Remark. Note that $\widehat{z}_{2}$ and $\widehat{z}_{3}$ do not converge to $z_{2}$ and $z_{3}$. In steady state $\left(z_{1}=\hat{z}_{1}=0\right), z_{2}-\widehat{z}_{2}$ and $z_{3}-\widehat{z}_{3}$ evolve as a harmonic oscillator.

For this example it is possible to use the reduced order dynamic controller from section 3.3 (or from $[\mathrm{CHP}]$ ) for the corresponding assumptions are satisfied. One can choose $Q_{2}=I_{2}$ to satisfy (32) and our controller (33)-(34) takes the form

$$
\begin{aligned}
u & =-z_{1}-z_{1} \widehat{z}_{2} \\
\dot{\widehat{z}}_{2} & =-\widehat{z}_{3}+z_{1} \phi\left(z_{1}\right) \\
\dot{\widehat{z}}_{3} & =\widehat{z}_{2} .
\end{aligned}
$$

It does yield boundedness of $\left(z_{1}, z_{2}, z_{3}, \hat{z}_{2}, \hat{z}_{3}\right)$ and convergence of $z_{1}$ to zero. One may again note that $z_{2}-\widehat{z}_{2}$ and $z_{3}-\widehat{z}_{3}$ oscillate when $z_{1}=0$.

Let us consider the methods from [IB] for this class of examples. The linear approximation of the system, i.e. the linear system $(F, G, H)$ with

$$
F=\left[\begin{array}{ccc}
0 & \phi(0) & 0 \\
0 & 0 & -1 \\
0 & 1 & 0
\end{array}\right], \quad G=\left[\begin{array}{l}
1 \\
0 \\
0
\end{array}\right], \quad H=[1,0,0]
$$

is detectable if and only if $\phi(0) \neq 0$. Our techniques work for any function $\phi$. If $\phi(0)=0$, for instance $\phi\left(z_{1}\right)=z_{1}$, the technique developed in [IB] does not apply. One may however notice that our controller is unnecessarily complex since, instead of the full-state feedback $u_{\text {nom }}$ given above, one may chose the static output feedback control $u=-z_{1}$ to globally stabilize $z_{1}$. If $\phi(0) \neq 0$, for instance $\phi\left(z_{1}\right)=1+z_{1}^{2}$, the technique developed in [IB] applies, but gives only local regulation; note that in this case there exists no static output feedback which regulates $z_{1}$.

Example 2. Consider the system

$$
\begin{aligned}
\dot{z}_{1} & =z_{1} z_{3}+z_{2} u \\
\dot{z}_{2} & =z_{1}-z_{3} \\
\dot{z}_{3} & =z_{2}-z_{1}^{2} \\
y & =z_{1} .
\end{aligned}
$$

which, again, is clearly of the form (1) with $x=\left(z_{1}, z_{2}, z_{3}\right), x_{1}=z_{1}, x_{2}=\left(z_{2}, z_{3}\right)$.

The interest of this example, compared to the previous one, is that it is not possible -or at least we have not been able- to build $u_{\text {nom }}$ and $V_{1}$ to meet the assumptions of section 3.3 (i.e. of $[\mathrm{CHP}])$, nor is it possible to find $u_{\text {nom }}$ and $V_{1}$ meeting assumption $\mathrm{A} 1$ or $\mathrm{A}^{\prime \prime}\left(x_{2}=0 \Rightarrow \dot{x}_{1}=0\right.$ whatever $u$ and $x_{1}$ are).

With $V_{1}=\frac{1}{2}\left(z_{1}^{2}+z_{2}^{2}+z_{3}^{2}\right)$ and $V_{2}=0$, we have

$$
\dot{V}_{1}=z_{1} z_{2}(1+u) .
$$

If we take $u_{\text {nom }}=-1-z_{1} z_{2}$ we obtain $\dot{V}_{1}=-z_{1}^{2} z_{2}^{2}$, which does not satisfy assumption A1, but a simple computation shows that assumption $\mathrm{A} 1^{\prime}$ is met. Assumption A2 is clearly satisfied, with $L=0$ and $M=I$. However our observability assumption A3' cannot be met. Indeed $Q(E-K H)+(E-K H)^{T} Q+N$ cannot be negative definite for any choice of $k_{1}, k_{2}, k_{3}$ since 
if it were, the $2 \times 2$ submatrix of it obtained by deleting the first row and column would also be negative definite. This submatrix is

$$
\left[\begin{array}{cc}
2\left(q_{12} u_{\text {nom }}+q_{23}\right) & q_{12} z_{1}+q_{13} u_{\text {nom }}+q_{33}-q_{22} \\
q_{12} z_{1}+q_{13} u_{\text {nom }}+q_{33}-q_{22} & 2\left(q_{13} z_{1}-q_{23}\right)
\end{array}\right],
$$

and if it were negative definite for all $z$, then the diagonal terms would be negative. Since $z_{1}$ and $u_{\text {nom }}$ may take any real values, this implies $q_{12}=q_{13}=0$, and the determinant of this submatrix would be $-4 q_{23}^{2}-\left(q_{33}-q_{22}\right)^{2}$, which is obviously negative.

On the other hand, the detectability assumption A3 is satisfied by choosing $k_{1}=1, k_{2}=u_{\text {nom }}$ and $k_{3}=z_{1}$, but we have no theorem for the case $\mathrm{A} 1^{\prime}+\mathrm{A} 2+\mathrm{A} 3$. Let us proceed to check that the more implicit but less restrictive assumption A4 is met.

The system (53) with the controller (33)-(34), using

$$
u=u_{\text {nom }}\left(z_{1}, \widehat{z}_{2}, \widehat{z}_{3}\right)=-1-z_{1} \widehat{z}_{2}
$$

becomes

$$
\begin{aligned}
& \dot{z}_{1}=z_{1} z_{3}-z_{2}\left(1+z_{1} \widehat{z}_{2}\right) \\
& \dot{z}_{2}=z_{1}-z_{3} \\
& \dot{z}_{3}=z_{2}-z_{1}^{2} \\
& \dot{\hat{z}}_{1}=-\widehat{z}_{1}+z_{1}\left(1+\widehat{z}_{3}-\widehat{z}_{2}^{2}\right)-\widehat{z}_{2} \\
& \dot{\widehat{z}}_{2}=-\widehat{z}_{3}+\widehat{z}_{1}-z_{1} \widehat{z}_{2}\left(2 z_{1}-\widehat{z}_{1}\right) \\
& \dot{\widehat{z}}_{3}=\widehat{z}_{2}-z_{1} \widehat{z}_{1}
\end{aligned}
$$

From equation (30) we have $\eta(z, \widehat{z})=\left(z_{1}-\widehat{z}_{1}\right)^{2}+z_{1}^{2} \widehat{z}_{2}^{2}$. It is straightforward to verify that if $\eta(z(t), \widehat{z}(t))=0 \quad \forall t$ and $(z(t), \widehat{z}(t))$ satisfies $(54)$, then $z_{1}(t)=0 \forall t$. Therefore assumption A4 is met and Theorem 2 implies that $z_{1} \longrightarrow 0$ and $z_{2}, z_{3}, \widehat{z}$ are bounded.

As remarked in Section 2, our restrictive assumptions are assumption A2 (restricting the form of $V_{1}$ ) and the fact that, in assumption A3 (or its variations) the matrix $Q$ is asked to be constant. The following example from [CHP] displays a situation where assumption A3 cannot be met by any constant matrix $Q$, but an extension of our methods using a dynamic $Q$ does allow stabilization of $z_{1}$.

Example 3. Consider the system $(k=1, \ell=2)$ defined by $(2)$ with

$$
\begin{aligned}
a_{1}\left(z_{1}, u\right)=u & A_{1}\left(z_{1}, u\right)=(0,1) \\
a_{2}\left(z_{1}, u\right)=\left[\begin{array}{c}
z_{1} \\
0
\end{array}\right] & A_{2}\left(z_{1}, u\right)=\left[\begin{array}{cc}
0 & 0 \\
2 z_{1} & 0
\end{array}\right] .
\end{aligned}
$$

Or, using for convenience $z_{2}=\left[\begin{array}{l}p \\ q\end{array}\right]$,

$$
\left\{\begin{array}{cl}
\dot{z}_{1} & =q+u \\
\dot{p} & =z_{1} \\
\dot{q} & =2 p z_{1} .
\end{array}\right.
$$

With

$$
\left\{\begin{aligned}
u_{\mathrm{nom}}(x) & =u_{\mathrm{nom}}\left(z_{1}, p, q\right)=-z_{1}-p-q \\
V_{1}(x) & =\frac{1}{2} z_{1}^{2}+\frac{1}{2} p^{2} \\
V_{2}(x) & =\left(p^{2}-q\right)^{2} \\
\rho(x) & =-z_{1}^{2} \\
U_{1}\left(z_{1}\right) & =\frac{1}{2} z_{1}^{2} \text { and } M=\left[\begin{array}{ll}
1 & 0 \\
0 & 0
\end{array}\right],
\end{aligned}\right.
$$


assumptions A1 and A2 are satisfied. On the other hand, neither assumption A3 nor even assumption A4 can be satisfied: there is no (constant) positive definite $Q$ such that

$$
Q(E-K H)+(E-K H)^{T} Q+N \leq 0 .
$$

This follows from the observation that the determinant of the $2 \times 2$ submatrix of (55) obtained by deleting the first row and column ( $K$ has no action on this submatrix) is negative for $z_{1}$ large enough whatever the constant entries of $Q$ are. Note that the assumption (32) cannot be satisfied either since it is a particular case of (55) forcing some entries of $Q$ to be zero.

Using any positive definite $Q_{2}$, the reduced order controller (33)-(34) (coming from [CHP]) becomes

$$
\begin{gathered}
u=u_{\mathrm{nom}}\left(z_{1}, \widehat{p}, \widehat{q}\right) \\
{\left[\begin{array}{c}
\dot{\hat{p}} \\
\dot{\hat{q}}
\end{array}\right]=Q_{2}^{-1}\left[\begin{array}{c}
z_{1} \\
z_{1}
\end{array}\right]+\left[\begin{array}{c}
z_{1} \\
2 \widehat{p} z_{1}
\end{array}\right]}
\end{gathered}
$$

and, following the proof of Theorem 1 but taking

$$
W_{1}=V_{1}\left(z_{1}, z_{2}\right)+\frac{1}{2} \tilde{z}_{2}^{T} Q_{2} \tilde{z}_{2} \quad \text { and } \quad W=V_{2}+W_{1}
$$

we obtain

$$
\dot{W} \leq-\rho\left(z_{1}, \widehat{p}, \widehat{q}\right)+\frac{1}{2}\left[\begin{array}{c}
p-\widehat{p} \\
q-\widehat{q}
\end{array}\right]^{T}\left(Q_{2} \widehat{A}_{2}+\widehat{A}_{2}^{T} Q_{2}\right)\left[\begin{array}{c}
p-\widehat{p} \\
q-\widehat{q}
\end{array}\right]
$$

Since $A 3^{\prime \prime}$ is not met, $W$ need not decrease along solutions of the closed-loop system. However, we can extend our method by using a dynamic $Q_{2}$ : instead of taking $Q_{2}$ constant, we take $Q_{2}(t)$ to satisfy

$$
\dot{Q}_{2}=-Q_{2} \widehat{A}_{2}-\widehat{A}_{2}^{T} Q_{2}
$$

with a positive definite initial condition $Q_{2}(0)$, so that $\dot{W} \leq \rho\left(z_{1}, \widehat{p}, \widehat{q}\right)$. Thus we can conclude that $\left(z_{1}, p, q, \widehat{p}, \widehat{q}\right)$ remain bounded and $z_{1} \rightarrow 0$ provided that $Q_{2}(t)$ remains bounded and bounded away from singular matrices. Since the bottom-right entry of $Q_{2} \widehat{A}_{2}+\widehat{A}_{2}^{T} Q_{2}$ is zero, the corresponding entry in $Q_{2}(t)$ is constant (we will take it to be 1). With $Q_{2}=\left[\begin{array}{cc}\lambda & \mu \\ \mu & 1\end{array}\right],(56)$ becomes

$$
\begin{aligned}
\dot{\mu} & =-2 z_{1} \\
\dot{\lambda} & =-4 z_{1} \mu
\end{aligned}
$$

and $W$ can be written as

$$
W=\frac{1}{2} z_{1}^{2}+\frac{1}{2} p^{2}+\left(p^{2}-q\right)^{2}+\frac{1}{2}\left[\left(\lambda-\mu^{2}\right)(p-\widehat{p})^{2}\right]+\frac{1}{2}[\mu(p-\widehat{p})+(q-\widehat{q})]^{2} .
$$

Therefore $z_{1}, p, q$, and the quantities in square brackets are bounded. Noting that $\lambda-\mu^{2}$ is constant we can conclude that $\widehat{p}$ is bounded. Since

$$
\frac{1}{2} \mu^{2}-\left(\lambda-\mu^{2}+1\right) \mu-2\left(\lambda-\mu^{2}\right) \widehat{p}
$$

is also constant we can conclude that $\mu$ is bounded, and therefore $\widehat{q}$ is bounded as well.

\section{Conclusions}

We have presented here some new methods for building output feedback controllers from full state feedback controllers for nonlinear systems. The use of Lyapunov based techniques allows us to obtain global results. Our methods do not rely on explicitly building an observer. On the contrary, we have considered a more direct approach to the problem of output feedback regulation as a whole. This is, in our opinion, the main contribution of this work. 


\section{Acknowledgments}

The authors wish to thank Laurent Praly for fruitful discussions on the subject.

\section{References}

[A] Artstein, Z., "Stabilization with relaxed controls", Nonl. Anal., TMA, Vol. 7, 1983.

[B] Brockett, R.W., "Finite Dimensional Linear Systems," Wiley, 1970.

[CHP] Cebuhar, W. A., Hirschorn, R. W. and Pomet, J.-B., "Some results on dynamic output feedback regulation of nonlinear systems," 30th CDC Conference, Brighton, England, 1991.

[F] Francis, B., "The linear multivariable regulator problem ", SIAM J. Contr. Opt., Vol. 15, No. 3, 1977 .

[GK] Gauthier, J.-P. and Kupka, I., "A separation principle for bilinear systems with dissipative drift", IEEE Trans. Auto. Control, Vol. AC-37, No. 12, pp 1970-1974, 1992.

[I] Isidori, A., "Nonlinear Control Systems, 2nd. Edition," Springer-Verlag, 1989.

[IB] Isidori, A. and Byrnes, C. I., "Output regulation of nonlinear systems", IEEE Trans. Auto. Control, Vol. AC-35, No. 2, 1990.

[JQ] Jurdjevic, V. and Quinn, J.P., "Controllability and Stability," Journal of Differential Equations, Vol. 28, 1978.

[KKM] Kanellakopoulos, I., Kokotovic, P.V. and Morse, A.S., "A toolkit for nonlinear feedback design," Systems and Control Letters, Vol. 18, pp. 83-92, 1992.

[L] LaSalle, J.-P., "Stability theory for ordinary differential equations," Journal of Differential Equations, Vol. 4, 1968.

[MT1] Marino, R. and Tomei, P., "Global adaptive observers and output feedback stabilization for a class of nonlinear systems," In: P.V. Kokotovic, Ed., Foundations of Adaptive Control, Springer-Verlag, pp. 455-493, 1991.

[MT2] Marino, R. and Tomei, P., "Output Feedback Control of a class of nonlinear systems," 2nd IFAC NonLinear Control Systems symposium, Bordeaux, 1992.

[NV] Nijmeier, H. and Van der Schaft, A.J., "Nonlinear Dynamical Control Systems," SpringerVerlag, 1990.

[P] Praly, L., "Lyapunov design of a dynamic output feedback for systems linear in their unmeasured state components," 2nd IFAC NonLinear Control Systems symposium, Bordeaux, 1992.

[PBPJ] Praly, L., Bastin, G., Pomet, J.-B. and Jiang, Z. P., "Adaptive Stabilization of Nonlinear Systems", to be published in the series: Lecture Notes in Information and Control, Springer-Verlag, 1991.

[S1] Sontag, E. D., "A universal construction of Artstein's theorem on nonlinear stabilization", Systems and Control Letters, Vol. 13, No. 3, 1989.

[S2] Sontag, E. D., "Conditions for Abstract Nonlinear Regulation", Information and Control, Vol. 51, No. 2, pp.105-127, 1981. 
[T] Tsinias, J., "A generalization of Vidyasagar's theorem on stabilizability using state detection," Systems and Control Letters, Vol. 17, No. 1, 1991.

[TK] Tsinias, J. and Kalouptsidis, N., "Output feedback stabilization", IEEE Trans. Auto. Control, Vol. AC-35, No. 8, 1990.

[V] Vidyasagar, M., "On the stabilization of nonlinear systems using state detection," IEEE Trans. Auto. Control, Vol. AC-25, June 1980. 